\title{
Sul termine cala nel senso di 'spazzaneve'
}

\author{
Bruno Moretti
}

PUBBLICATO: 23 FEBBRAIO 2021

\section{Quesito:}

Un docente di liceo, che si descrive come "lombardo di origine e sempre vissuto in Lombardia" segnala di essersi accorto con sorpresa del fatto che la parola cala, nel significato di 'spazzaneve', non è nota al di fuori della Regione. Vale la pena di riportare quasi per intero la sua formulazione: "Stupito perché per trentacinque anni mi era parsa una parola italiana standard, ho cercato notizie [...]; ho trovato solo due articoli che la registrano come parola ticinese. Ora, effettivamente vivo molto vicino al confine, e da sempre mi è chiaro il confronto con l'italiano parlato in Svizzera: percepisco alcune forme come marcatamente ticinesi, ma non questa. Esistono studi che siano in grado di ricostruire la storia della parola, se sia effettivamente ticinese e passata nell'italiano delle zone di confine o, viceversa, propria dei dialetti lombardi occidentali in genere e non solo di quelli ticinesi?"

\section{Sul termine cala nel senso di 'spazzaneve'}

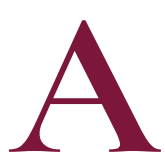

bbiamo voluto riportare in modo esteso questa citazione perché mostra molto bene come per i parlanti le soluzioni linguistiche imparate da bambini e usate per tutta la vita non siano percepite come regionali fino al momento in cui le persone non diventano consapevoli del fatto che parlanti di altre regioni non conoscono le forme in questione o quando si accorgono che esse non fanno parte dello standard normativo. In breve, prima che nasca la consapevolezza della regionalità specifica, per i parlanti queste non sono forme regionali, ma forme "normali".

Va inoltre notato che una parte molto importante di queste peculiarità dei singoli italiani regionali sono riprese dai dialetti, dove spesso hanno continuato un etimo latino che potenzialmente anche l'italiano normativo, o altri italiani regionali, avrebbero potuto sfruttare con lo stesso esito. In questo senso molte soluzioni lessicali regionali derivate dai dialetti possono essere definite come "forme possibili" dell'italiano standard, che quest'ultima varietà non ha adottato allo stesso modo dei dialetti o dei vari italiani regionali, ma che non sono in contrasto con le regole dell'italiano.

Questo esempio specifico va pure a toccare il problema del confine nazionale tra Svizzera e Italia e ci darebbe la possibilità di aprire un lungo discorso, che qui possiamo solo accennare, sul ruolo dei confini politici tra Stati nazionali che condividono la stessa lingua e sul cosiddetto "policentrismo", ovvero il fatto che una stessa lingua possa presentare fenomeni di standardizzazione differenti in nazioni differenti (basti pensare al caso dell'inglese della Gran Bretagna, contrapposto a quello degli Stati Uniti o dell'Australia). Mentre una forma usata per esempio a Varese, deve fare i conti con le altre soluzioni usate nella Regione Lombardia e nel resto d'Italia, per una forma usata nel Canton Ticino la pressione esercitata dalle scelte linguistiche italiane è molto meno rilevante, ma conteranno molto di più le norme esplicite e implicite dell'amministrazione svizzera e di altri canali di standardizzazione. Più una forma riuscirà a entrare in una varietà formale alta e scritta, più acquisirà un valore normativo ufficiale. Nel caso degli standard d'Italia e di Svizzera le differenze sono indubbiamente assai ridotte, ma ciononostante, con argomenti validi, vari studiosi (come Elena Maria Pandolfi o Gaetano Berruto) sostengono che si possa attribuire anche all'italiano lo statuto di "lingua policentrica". 
Che cosa vuol dire ciò nel caso di cala per spazzaneve? Chiunque faccia una semplice ricerca in Internet dicallaneve o calla neve (con in entrambi i casi il raddoppiamento della consonante intervocalica tipico dell'italianizzazione di parole dialettali settentrionali) scoprirà tutta una serie di pagine web ufficiali di amministrazioni comunali della Svizzera italiana (quindi non solo del Canton Ticino, ma anche delle valli italofone del Canton Grigioni) che informano sul servizio di sgombero della neve, su concorsi ufficiali per l'assegnazione dello stesso, oppure pagine di quotidiani e altri mass media che si servono di queste espressioni per discutere di problemi legati allo sgombero della neve. Nel nostro caso specifico possiamo dire che l'uso ufficiale attuale dell'espressione si è fondamentalmente fermato a questo livello delle amministrazioni comunali e di pubblicazioni locali, dato che, con un rapido controllo, non è stato possibile riscontrarlo in pagine ufficiali dell'amministrazione cantonale (dove non costituisce quindi la norma) e pure nelle pagine dell'amministrazione federale si ritrova solo spazzaneve.

Venendo ora in modo più specifico alle domande del lettore, abbiamo la fortuna di poter rispondere senza dover fare grandi ricerche ma rifacendoci alla più che eccellente trattazione del lemma cala' fatta da Dario Petrini nel Vocabolario dei dialetti della Svizzera italiana (VSI; per gli abbonati è consultabile online).

Innanzitutto Petrini ci dice che il lemma, di genere femminile, ha due significati: "ı. via aperta nella neve; 2. spazzaneve". Nella prima accezione si ritrovano nelle differenti varietà dialettali della Svizzera italiana usi come "sentiero che si apre spalando la neve", "strada tagliata nella neve", "solco aperto dallo spazzaneve", "pestare la neve coi piedi per aprire la via". Petrini sintetizza dicendo che "in genere, vale ripulire le vie dalla neve, in particolare quelle di una certa importanza, con mezzi diversi (pale, badili, attrezzi appositi trainati)."

Le attestazioni riportate risalgono almeno fino al XVI secolo e ci ricordano pure che in molti casi "Lo sgombero della neve dalle strade era un lavoro da svolgere in comune". Rinunciando qui a riportare le indicazioni delle fonti, per le quali rimando alla scheda di Petrini, vale la pena di riportare anche le seguenti citazioni, che ricordano appunto il modo in cui il funzionamento di alcune comunità era organizzato nel passato: "quando scendevano almeno quindici $\mathrm{cm}$ di neve suonavano le campane tre volte a doppio, e un membro per famiglia andava sul piazzale davanti alla casa comunale dove si faceva l'appello"; "chi non poteva andare era tenuto a incaricare un sostituto, e se non si presentava nessuno si riceveva una multa".

Il secondo significato ('spazzaneve') è quello che ci interessa più specificamente. Anche in questo caso basta citare Petrini (escludendo i riferimenti specifici ai luoghi in cui si sono raccolte le varie espressioni e traducendo le stesse dal dialetto all'italiano):

Dall'uso di apposite grandi slitte di forma triangolare, dette anche slitta da cala, macchina della cala, cassone della cala, composte di due assi ad angolo unite fra loro con una stanga, e attaccate al loro vertice a un mezzo di traino (cavalli, buoi, in seguito veicoli a motore), più recentemente spinte avanti da autoveicoli, cala viene a designare lo spazzaneve, destinato in genere a sgomberare le strade del comune: cala, strumento che serve ad aprire un varco nella neve, slitta per levare la neve dalle strade.

Dal dialetto, come si è detto, l'espressione passa allitaliano regionale, dove manifesta tutt'ora una buona vitalità nella Svizzera italiana e, come abbiamo visto, compare anche in usi scritti.

Dall'espressione fare la cala si sono poi sviluppati anche sensi traslati, come quello che rimanda in modo scherzoso a "spazzare tutto ciò che rimane da mangiare in tavola" o a "spianare la via a qualcuno". 
I due significati ('via aperta nella neve' e 'spazzaneve') sono indubbiamente legati tra loro da un rapporto che possiamo definire come metonimico. Petrini lo illustra nel modo seguente:

In questa prospettiva, il significato (moderno) di "spazzaneve" può sorgere da quello di "sentiero", grazie al comparire di cala nella locuzione fare la cala, a un certo punto non più analizzata nel suo significato primario di "fare il sentiero", ma intesa come "sgomberare dalla neve" (in cui può avere eventualmente giocato la presenza di calá "(far) diminuire"), donde, ancora, attraverso formazioni del tipo slitta, macchina, uomini ecc. della cala "attrezzi, persone con la funzione specifica di sgomberare dalla neve", si ebbe infine cala "spazzaneve".

Per quanto concerne l'origine etimologica e la diffusione geografica, Petrini rimanda al latino callis 'strada, sentiero' (ritrovabile come calle nell'italiano letterario o, per esempio, nei dialetti veneti: basti pensare tipicamente ai nomi delle vie veneziane) e segnala che l'area ticinese (e della valle Anzasca) continua a est in Valtellina, nella Bergamasca e nel Bresciano. Fonti ritrovabili in Internet, ma la cui affidabilità è difficile da verificare, segnalano l'uso di cala con il valore di 'spazzaneve' anche in Provincia di Novara e di Asti.

Non in tutte le zone e per tutti i parlanti l'espressione sembra essersi trasmessa stabilmente dal dialetto allitaliano regionale. È significativo per esempio che tra i parlanti italiani ai quali Petralli sottopone varie forme di italiano regionale ticinese (per la sua indagine pubblicata nel I990 con il titolo L'italiano in un cantone) solo l'informatore di Varese dichiari di conoscerla, connotandola però come un termine dialettale e arcaico. Gli informatori di Bergamo, Milano, Sondrio, Novara e Bologna dicono invece che non si usa.

A ovest e a sud dell'area di diffusione di cala si ritrova invece la forma calata dei dialetti liguri, piemontesi, lombardo occidentali (anche in questo caso rinvio per l'accurata documentazione delle fonti a Petrini). A fare la cala corrisponde in queste zone fare la calata o passare la calata (in quest'ultimo caso il valore di calata è quello dell'attrezzo impiegato, ovvero un triangolo di legno appesantito e trainato che spingeva la neve ai lati).

Per quanto riguarda l'origine di calata, Petrini accenna al fatto che questa forma presupporrebbe l'esistenza di un verbo denominale italiano settentrionale *allare, che non è però attestato in quest'area, ma si ritrova comunque nell'area francoprovenzale (con il valore di 'aprirsi un sentiero nella neve'). L'eventuale esistenza di "callare potrebbe spiegare in modo alternativo a quanto fatto sopra l'origine di cala per 'spazzaneve' (che sarebbe allora un deverbale senza suffisso) e altre forme dialettali come calin o caladora 'spazzaneve', o caladoo 'addetto allo spazzaneve'.

Concludiamo segnalando uno strumento che in futuro si potrà rivelare molto utile (se avrà la risonanza che si spera) per studiare la diffusione attuale di varie espressioni regionali. Si tratta di un progetto (e di uno strumento di divulgazione sociolinguistica) avviato sotto la responsabilità scientifica dell'Osservatorio linguistico della Svizzera italiana, che mira a raccogliere dati geolinguistici (che poi vengono resi visibili trasformandoli in mappe) attraverso la partecipazione spontanea di persone di lingua italiana, residenti in differenti regioni, che possono fornire direttamente i dati rispondendo ad una serie di domande. Chi fosse interessato, trova tutte le informazioni al seguente indirizzo www.lidatè.ch (va aggiunto che i dati relativi al nome dello 'spazzaneve' però non sono ancora stati raccolti). 


\section{Cita come:}

Bruno Moretti, Sul termine cala nel senso di 'spazzaneve', "Italiano digitale", 2021, XVI, 2021/1 (gennaio-marzo)

DOI: $10.35948 / 2532-9006 / 2021.5479$

Copyright 2021 Accademia della Crusca

Pubblicato con licenza creative commons CC BY-NC-ND 